OPEN ACCESS

Edited by:

Wei Zhao,

City University of Hong Kong,

Hong Kong

Reviewed by:

Peng Shen,

Nanjing Medical University, China

Xiancheng Liu,

Affiliated Hospital of Nantong

University, China

*Correspondence:

Yaozhen Pan

panyaozhen112@163.com

${ }^{\dagger}$ These authors have contributed equally to this work

Specialty section:

This article was submitted to Molecular and Cellular Oncology,

a section of the journal

Frontiers in Cell and Developmental

Biology

Received: 31 March 2021

Accepted: 21 June 2021

Published: 27 July 2021

Citation:

Chen T, Huang $B$ and Pan $Y$ (2021) Long Non-coding RNA

MAFG-AS1 Promotes Cell Proliferation, Migration, and EMT by miR-3196/STRN4 in Drug-Resistant

Cells of Liver Cancer.

Front. Cell Dev. Biol. 9:688603. doi: 10.3389/fcell.2021.688603

\section{Long Non-coding RNA MAFG-AS1 Promotes Cell Proliferation, Migration, and EMT by miR-3196/STRN4 in Drug-Resistant Cells of Liver Cancer}

\author{
Tianming Chen ${ }^{1+}$, Bin Huang ${ }^{2+}$ and Yaozhen Pan ${ }^{3 *}$ \\ ${ }^{1}$ Department of Surgery, Drum Tower Hospital Affiliated to Nanjing University Medical School, Nanjing, China, ${ }^{2}$ The \\ Comprehensive Cancer Center of Nanjing Drum Tower Hospital, The Affiliated Hospital of Nanjing University Medical School \\ \& Clinical Cancer Institute of Nanjing University, Nanjing, China, ${ }^{3}$ Department of Hepatobiliary Surgery, The Affiliated Hospital \\ of Guizhou Medical University, Guiyang, China
}

Long non-coding RNAs (IncRNAs) have been shown to participate in the development and progression of several different types of cancer. Past studies indicated that IncRNA MAFG-antisense 1 (AS1) promotes colorectal cancer. However, the role of MAFG-AS1 in hepatocellular carcinoma $(\mathrm{HCC})$ remains unclear. The aim of the present study is to examine the effect of IncRNA MAFG-AS1 on drug resistance HCC. The results indicated that MAFG-AS1 is upregulated in drug-resistant cells. Further, MAFG-AS1 promotes growth and migration of HCC by upregulating STRN4 through absorbing miR-3196. Thus, LncRNA MAFA-AS1 may become a novel target to treat HCC patients.

Keywords: long non-coding RNA, MAFG-AS1, miR-3196, STRN4, liver cancer

\section{INTRODUCTION}

Liver cancer is the sixth most ordinary cancer and the third main cause of death of cancer (Altekruse et al., 2014). Liver cancer could be induced by many factors, including hepatitis B virus, alcohol abuse, aflatoxin, and hepatitis $\mathrm{C}$ virus infection; non-alcoholic fatty liver disease could also increase the risk of liver cancer specifically (Duan et al., 2014). Although many advances have been achieved in the treatment of liver cancer, the survival rate is still low. Hepatocellular carcinoma (HCC) is proved to be the most common form of liver cancer, taking up 90\% of all liver cancers (Feng and Ho, 2014). HCC is featured by rapid growth and invasive tumor and has a tendency for a high probability of metastasis and recurrence (Dika and Abou-Alfa, 2017; Liu et al., 2017). Traditional therapeutic methods for HCC, including surgical resection and chemoradiotherapy, are hard to completely inhibit the tumor growth (Innes et al., 2018). Fundamentally, the molecular mechanisms for HCC metastasis need to be sequentially explored.

Long non-coding RNAs (lncRNAs) are classes of RNAs which are over 200 nt in length without protein-coding ability. LncRNAs can regulate important tumor biological functions, such as epithelial-mesenchymal transition (EMT), invasion-metastasis cascade, proliferation, and drug resistance (Yuan et al., 2014; Li et al., 2016; Tan et al., 2017; Xu et al., 2017). For instance, lncRNAATB facilitated cell proliferation in gastric cancer by miR-141-3p/TGF 32 signaling (Lei et al., 2017). Another study indicated that LncRNA Igf2 was upregulated in HCC cells and tissues and 
controlled HCC progression through the ERK/MAPK signaling pathway (Bao et al., 2017). Another example is that MAFb ZIP transcription factor G antisense RNA 1 (MAFG-AS1) can expedite cell proliferation and invasion in colorectal cancer by targeting miR-147b/NDUFA4 (Cui et al., 2018). Besides, it is reported that LINC00511 boosted the progression of breast cancer by targeting miR-185-3p/E2F1/Nanog ( $\mathrm{Lu}$ et al., 2018). MAFG-AS1 is a novel clinical biomarker for clinical progression and unfavorable prognosis in gastric cancer (Li et al., 2020). Downregulation of MAFG-AS1 represses tumorigenesis of colorectal cancer cells through the microRNA-149-3p-dependent inhibition of HOXB8 (Ruan et al., 2020).

However, few studies have provided quantitative evidence of the effects of MAFG-AS1 on HCC progression. Here, we studied the biological functions of MAFG-AS1 in HCC. The impacts of MAFG-AS1 on the regulation of cell invasion, migration, and EMT were also studied. These results provide important evidence that MAFG-AS1 may be a novel therapeutic target and a biomarker for predicting response to sorafenib treatment of HCC.

\section{MATERIALS AND METHODS}

\section{HCC Cell Lines}

Human HCC cell lines (Huh7, HepG2) and normal liver cell lines (Lo-2) were provided by the Chinese Academy of Sciences Cell Bank (Shanghai, China). In a humidified atmosphere with $5 \% \mathrm{CO}_{2}$, HCC cells were cultured in Dulbecco's modified Eagle's medium (DMEM, Gibco, Grand Island, NY, United States) with $10 \%$ fetal bovine serum (FBS, Gibco, Grand Island, NY, United States) at $37^{\circ} \mathrm{C}$. The related sorafenib-resistant cell line (HepG2/SF) was generated by exposing cells to increasing concentrations $(\leq 2 \mu \mathrm{M})$ of sorafenib.

\section{Chemicals}

The media and antibiotics for cell culture were purchased from HiMedia (Chandigarh, India), Sigma-Aldrich (St. Louis, MO, United States), and Thermo Fisher Scientific China (Shanghai, China). Sorafenib was procured from Santa Cruz Biotechnology, Inc. (Dallas, TX, United States). Sorafenib was dissolved in dimethyl sulfoxide (DMSO) to prepare 1- and 20-mM stocks, respectively, for further use in cell lines.

\section{Western Blotting}

Western blotting was performed according to a standard protocol. Total protein extracts from HCC cells transfected with miR-3196 mimics or miR-3196 inhibitor were loaded on SDS-PAGE gels and transferred into PVDF membranes. After being blocked by skimmed milk, these bands were incubated with primary antibodies at $4^{\circ} \mathrm{C}$ overnights. Next, membranes were washed with PBS followed by incubation with the appropriate secondary antibody for $1 \mathrm{~h}$ at $22-23^{\circ} \mathrm{C}$. Finally, enhanced chemiluminescence (ECL, Pierce, Rockford, IL, United States) was added to visualize these membranes. The mouse anti-E cadherin, vimentin, and $\alpha$-SMA antibodies (R\&D Systems Europe Ltd.) were diluted 500 times. Quantification of Western blotting was performed by densitometry using the Storm 820 Phosphor Imager (Molecular Dynamics, Sunnyvale, CA, United States).

\section{Real-Time Quantitative Polymerase Chain Reaction (qRT-PCR)}

qRT-PCR was performed as previously reported (Zhang et al., 2011). Total RNA was extracted with TRIzol. The primers used for gene amplification from the cDNA templates were as follows: $\alpha$-SMA: 5'-CTGACAGAGGCACCACTGAA-3, 5-CATCTCCAGAGTCCAGCACA-3'; MAFG-AS1: $\quad 5^{\prime}$-CGT TCT TAG TTG GTG GAG CG-3' and reverse, 5'-CCG GAC ATC TAA GGG CAT CA-3'; GAPDH: 5'-AATGG ATTTGGACGCATTGGT-3' ${ }^{\prime}$ 5' -TTTGCACTGGTACGTGTTG AT-3'. For miRNA, 800 ng RNA was transcribed for cDNA with a reverse transcription kit and miRNA-specific primers supplied by Clontech (Mir-X ${ }^{\mathrm{TM}}$ miRNA First-Strand Synthesis, San Francisco Bay, CA, United States). qRT-PCR was performed with miScript SYBR Green PCR Kit, and U6 was used as a normalized control. mouse-miR-3196 mimics, mouse-miR-3196 inhibitors, and their corresponding negative controls were purchased from Qiagen (Dusseldorf, Germany).

\section{Cell Counting Kit-8 (CCK-8) Assay}

Cell proliferation assay was performed according to the instruction of the CCK-8 kit (Solarbio, Beijing, China). Cells at the logarithmic phase were made into single-cell suspension and seeded to 96 -well plates with $5 \times 10^{3}$ cells. After seeding, $10 \mu \mathrm{l}$ of CCK-8 solution mixed with $90 \mu \mathrm{l}$ of DMEM was added into each well. After $2 \mathrm{~h}$ of incubation, absorbance was measured at $450 \mathrm{~nm}$. The 50\% growth inhibition (IC50) was measured according to a previous paper (Ma et al., 2017).

\section{Transwell Invasion and Migration Assay}

Transwell assays were performed as described previously (Wang et al., 2017). Briefly, Huh7/R and HepG2/R cells were seeded in Matrigel-coated upper chambers with a pore (50 lL Matrigel, BD Bioscience, United States). Medium without serum and $10 \%$ FBS was added into the upper chamber, and medium with $10 \%$ FBS was added into the lower chambers. After being incubated for $24 \mathrm{~h}$, the migrated and invaded cells on the lower membrane surface were fixed and then stained with $20 \%$ Giemsa solution. Five random fields were counted per chamber by an inverted microscope (Olympus, Japan). Each experiment was repeated three times.

\section{Wound Healing Assay}

Wound healing tests were used to evaluate cell migration in HCCs. Cells were transfected with $80 \mathrm{nmol} / \mathrm{l} \mathrm{miR}-3196$ mimics, or $80 \mathrm{nmol} / \mathrm{l} \mathrm{miR}-3196$ inhibitors, respectively. Cells are inoculated to produce confluent monolayers in a sixwell plate. The fused cells were scraped with a $200-\mu 1$ sterile pipette. After $48 \mathrm{~h}$ of cultivation, the wounds were evaluated using a microscope. 


\section{Immunohistochemistry Staining Analysis}

$\alpha$-Smooth muscle actin ( $\alpha$-SMA) was determined by immunohistochemistry staining. Briefly, sections were dewaxed and endogenous peroxidase activity was quenched by $3 \%$ hydrogen peroxide for $15 \mathrm{~min}$. Then, the mixture was blocked with normal goat serum for $30 \mathrm{~min}$ to eliminate non-specific binding and incubated overnight at $4^{\circ} \mathrm{C}$ with primary antibodies against $\alpha$-SMA (1:100; Abcam). The next day, the specific sections of the sample were treated with a PV6000 Histostain ${ }^{\mathrm{TM}}$ kit (ZSGB, Beijing, China) and stained with diaminobenzidine (ZSGB, Beijing, China). Finally, the sections were counterstained by hematoxylin and images acquired with FSX100 (Olympus, Tokyo, Japan).

\section{Luciferase Reporter Assay}

The $3^{\prime}$ untranslated region (UTR) fragments of STRN4 containing the miR-binding sites were amplified by PCR using the cDNA template obtained from the RNA sample of macrophages. The wild-type $3^{\prime}$ UTRs of STRN4 as well as mutant $3^{\prime}$ UTRs with nucleotide substitutions in the putative binding sites corresponding to the seed sequence of miR-3196 were cloned downstream of the firefly luciferase gene in the pGL3 vector (Promega, Madison, WI, United States). Cells were co-transfected with miR-3196 or a control microRNA. Fortyeight hours later, cells were washed in PBS and luciferase activity was measured by a luminometer (Promega, Madison, WI, United States), using a dual-luciferase reporter assay system.

\section{RNA Immunoprecipitation (RIP)}

RIP was performed using the Magna RIP RNA-Binding Protein Immunoprecipitation Kit (Sigma, CA, United States). HepG2 was transfected with miR-3196-biotin or non-sense control (NC)biotin. Cells were lysed in RIP lysis buffer. A/G magnetic beads with anti-biotin ligation were used to pull down the miR-3196biotin immunoprecipitation. After the antibody was recovered by protein beads, qRT-PCR was performed to detect STRN4 and miR-3196 in the precipitates.

\section{RNA Fluorescence in situ Hybridization}

Fluorescence in situ Hybridization (FISH) assay was conducted, referring to the protocol of the kit from GenePharma (Shanghai, China).

\section{Statistical Analysis}

All data were analyzed using SPSS 17.0 software (IBM, Armonk, NY, United States); every experiment was repeated in triplicate, and the data were presented as mean \pm standard error (SE) of the mean. Statistical analysis was performed by Student's $t$-tests. Differences in more than three groups were determined by oneway analysis of variance (ANOVA) test followed by

\section{A}

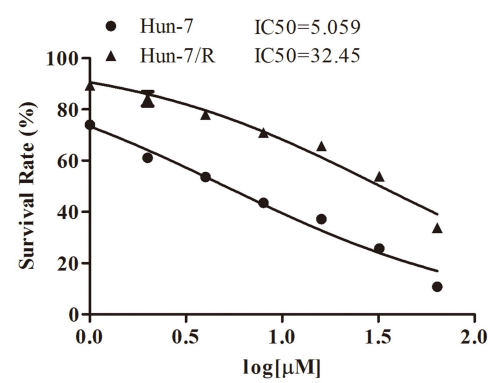

C

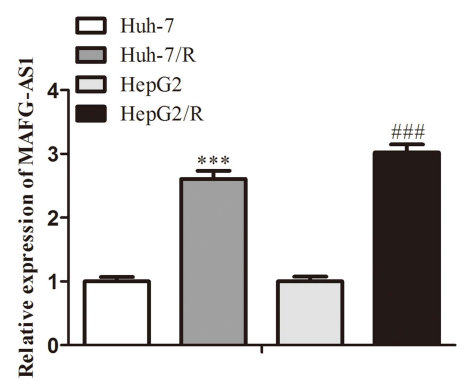

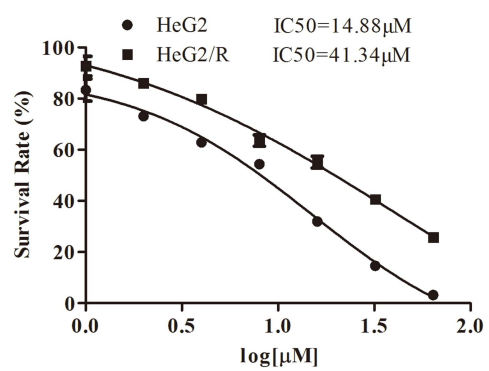

D

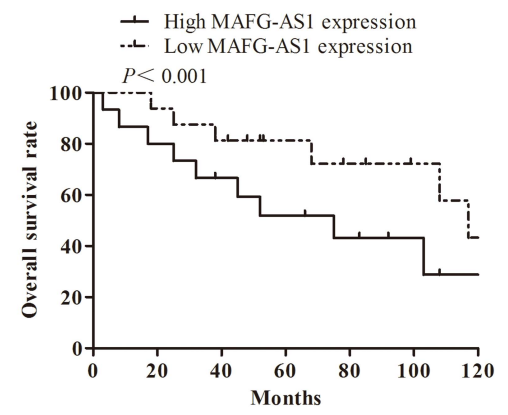

FIGURE 1 | A high expression of MAFG-AS1 is found in drug-resistant cells of liver cancer. (A,B) The half-maximal inhibitory concentration (IC50) values of two liver cancer cell lines. (C) RT-PCR showed that MAFG-AS1 was upregulated in drug-resistant HCC cell lines (Huh7/R, HepG2/R) compared with parental cell lines. (D) The line chart showed the overall survival rate of HCC cell lines with different MAFG-AS1 expression levels. 
A

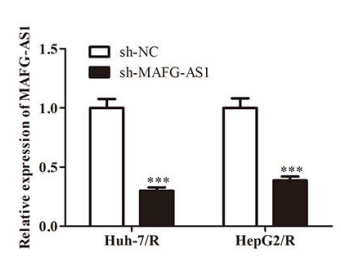

B
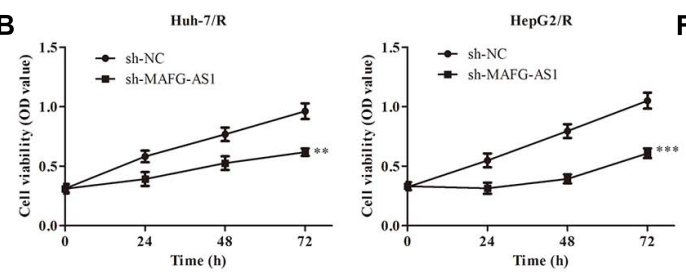

C

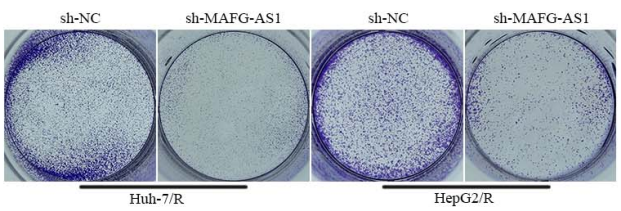

D
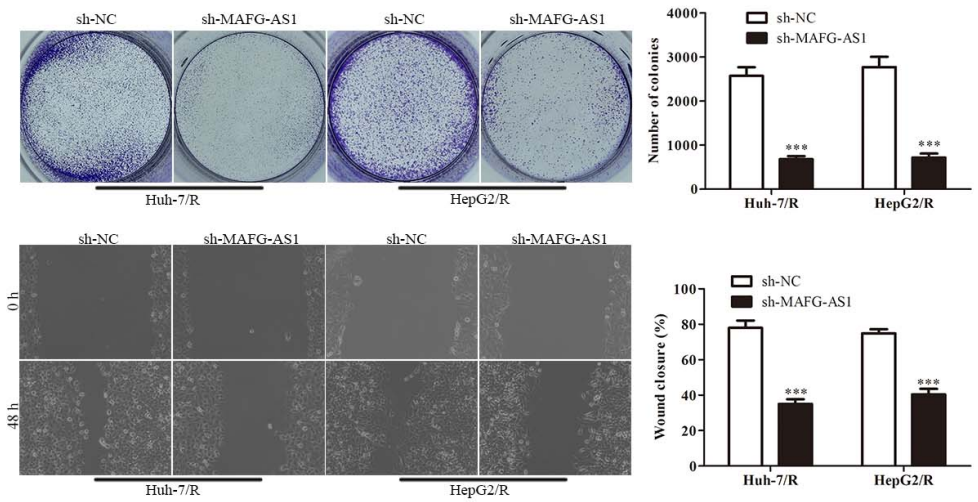

E

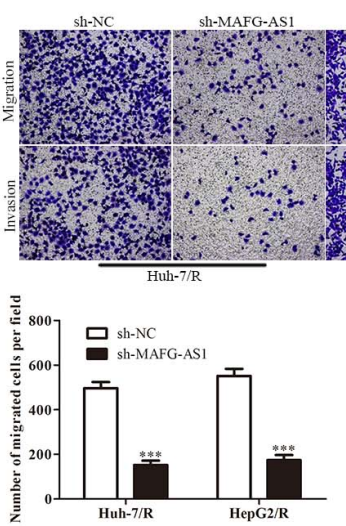

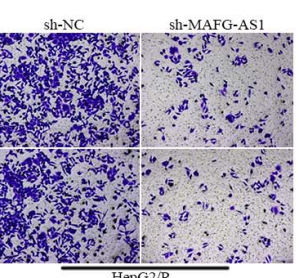

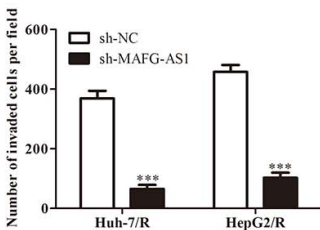

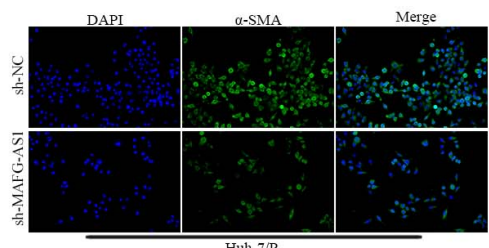

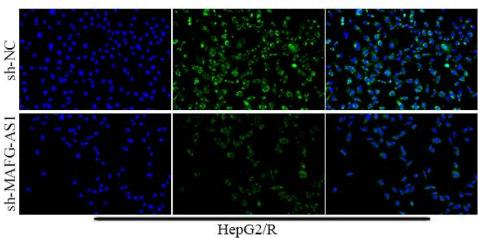

G
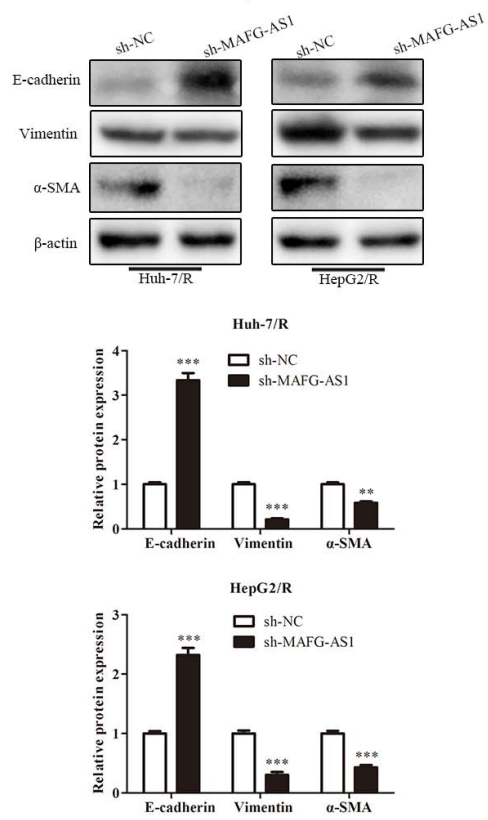

FIGURE 2 | MAFG-AS1 knockdown inhibits proliferation, invasion, migration, and EMT of HCC cells. (A) RT-PCR analysis was used to verify the interfering efficiency. (B) CCK-8 assay indicated the cell viability in the sh-MAFG-AS1 group compared with the sh-NC group. (C) The clone formation abilities of the Huh7/R and HepG2/R cells. (D,E) Wound healing assay (D) and transwell analysis $\mathbf{( E )}$ to test the effect on the invasion and migration of HCC cells. (F,G) Immunostaining (F) and western blotting (G) detected the EMT of Huh7/R and HepG2/R cells. Scale bars: $50 \mu \mathrm{m}$. ${ }^{\star \star} P<0.01,{ }^{* \star *} P<0.001$ vs sh-NC group.

Tukey's multiple comparison test. $p<0.05$ was considered statistically significant.

\section{RESULTS}

\section{High Expression of MAFG-AS1 Is Found in Drug-Resistant Cells of Liver Cancer}

To investigate the resistance of induced drug-resistant cells, we investigated the cytotoxicity by 3-(4,5-dimethylthiazol-2yl)-2,5-diphenyl tetrazolium bromide (MTT) assay. After a 48-h treatment with drugs, the growth of HCC cells was markedly inhibited in a concentration-dependent manner. The half-maximal inhibitory concentration (IC50) values of the drug ranged from 5 to $40 \mu \mathrm{M}$ for two liver cancer cell lines, respectively (Figures 1A,B). Meanwhile, expressions of
MAFG-AS1 in drug-resistant cell lines HepG2/R and Huh7/R and parent strain cells HepG2 and Huh7 were detected by RT-qPCR. The results showed that MAFG-AS1 expression in drug-resistant cell lines was obviously higher than that in normal strain cells (Figure 1C). Additionally, the HCC cells showed a longer overall survival rate with a low MAFG-AS1 expression (Figure 1D).

\section{MAFG-AS1 Knockdown Inhibits Proliferation, Invasion, Migration, and EMT of HCC Cells}

To further explore the effect of MAFG-AS1 on drug-resistant liver cancer cell lines, two low-expression MAFG-AS1-resistant cell lines were constructed, and the efficiency was detected by qRT-PCR (Figure 2A). CCK-8 assay was used to detect the viability of cells in each group. The results showed that in 
A

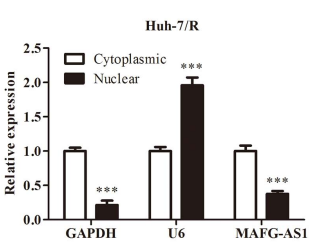

D

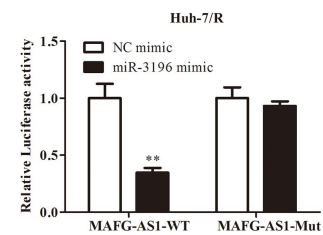

F

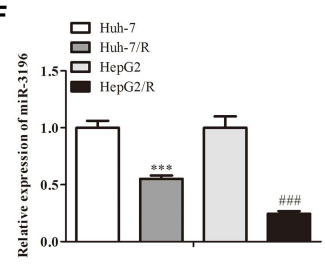

B
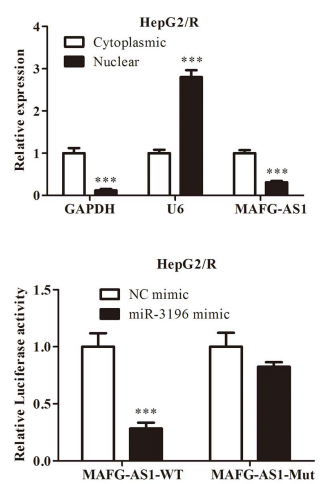

G

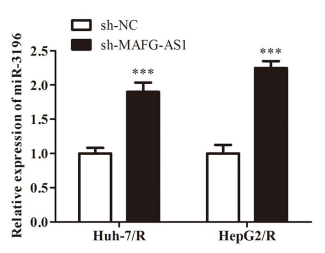

E
C

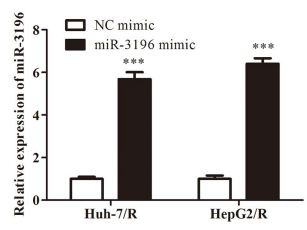

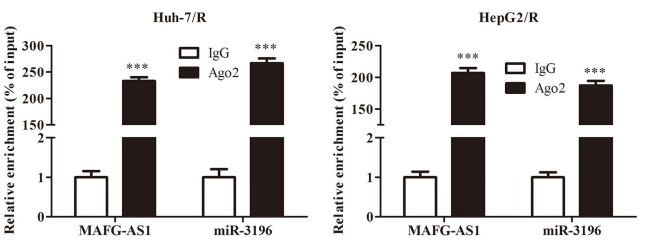

H

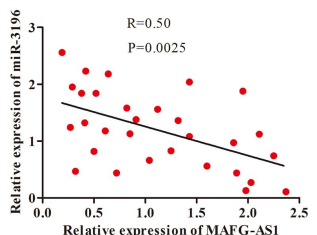

FIGURE 3 | miR-3196 bound with MAFG-AS1. (A) RNA-FISH assay implied the location of MAFG-AS1. ${ }^{\star \star *} P<0.001$ vs cytoplasmic group. (B) Schematic diagram demonstrated the complementarybinding within miR-3196 and MAFG-AS1 3'-UTR with binding sites predicted by bioinformatics programs (StarBase V 2.0, http://starbase.sysu.edu.cn/mirLncRNA). (C) RT-PCR analysis was used to verify the efficiency. ${ }^{* \star \star} P<0.001$ vs NC-mimic group. (D) Lucife rase reporter gene assay was performed in Huh7/R and HepG2/R cells transfected with MAFG-AS1 wild/mutant type and miR-3196 mimics/control. ${ }^{* \star} P<0.01$, ${ }^{\star \star \star} P<0.001$ vs NC-mimic group. (E) The relationship between MAFG-AS1 and Ago2 was detected by RIP assay. ${ }^{* \star *} P<0.001$ vs lgG group. (F) miR-3196 expression levels were measured in drug-resistant cell lines and parental cell lines using RT-PCR. ${ }^{* \star *} P<0.001$, \#\#\# $P<0.001$ vs Corresponding control group. (G) RT-PCR showed the miR-3196 expression levels in Huh7/R and HepG2/R cells transfected with MAFG-AS1 shRNA or empty vector. ${ }^{\star \star \star} P<0.001$ vs sh-NC group. (H) Pearson's correlation analysis of the correlation between miR-3196 and MAFG-AS1.

contrast to the sh-NC group, the viability of Huh7/R cells in the sh-MAFG-AS1 group was dramatically decreased. Meanwhile, the cell viability was greatly decreased in the sh-MAFG-AS1 group of HepG2/R cell lines (Figure 2B). To further confirm the inhibition of cell proliferation by sh-MAFG-AS1 in liver cancer cells, the colony formation assay and soft agar colony formation assay were conducted on Huh7/R and HepG2/R cells. As shown in Figure 2C, the clone formation abilities of the cells were clearly suppressed by incubation of MAFG-AS1. Next, we performed wound healing analysis to test the effect of MAFG-AS1 on the invasion and migration of HCC cells. The results showed that MAFG-AS1 knockdown significantly reversed liver cancer cell migration (Figure 2D). Moreover, transwell assay showed that in Huh7/R and HepG2/R cells, MAFG-AS1 knockdown decreased the migrated and invasive cell number compared to empty vector-transfected cells (Figure 2E). It has been reported in a previous study that EMT is a critical step of HCC metastasis (Ke et al., 2011). Next, we investigated whether MAFG-AS1 could induce EMT in HCC cells. The results showed that knockdown of MAFG-AS1 could significantly increase the expression of E-cadherin but decrease the expression of vimentin and $\alpha$-SMA in Huh7/R and HepG2/R cells, as demonstrated by IF or Western blotting (Figures 2F,G). Overall, these results concluded that MAFG-AS1 knockdown inhibited the proliferation, migration, invasion, and tumor growth of drug-resistant HCC cells in vitro, suggesting the potential tumor-promoting role of MAFG-AS1 in drug resistance of HCC.

\section{miR-3196 Bounds With MAFG-AS1}

To explore the mechanism of MAFG-AS1, RNA-FISH assay results were collected and analyzed, which implied that MAFGAS1 was indeed concentrated in the cytoplasm, indicating that MAFG-AS1 might play a role in the cytoplasm (Figure 3A). To investigate the potential molecular mechanism of MAFGAS1, bioinformatics prediction tools (StarBase V 2.0) ${ }^{1}$ were used. Results revealed that miR-3196 shared a complementary binding of MAFG-AS1 by binding sites (Figure 3B). To further explore the effect of miR-3196 on drug-resistant liver cancer cell lines, two miR-3196 mimic cell lines were constructed, and the efficiency was detected by qRT-PCR (Figure 3C). Furthermore, luciferase reporter gene assay showed that the luciferase activity was decreased in the combination of MAFGAS1 wild-type and miR-3196 mimics of Huh7/R and HepG2/R cells (Figure 3D). The relationship between MAFG-AS1 and Ago2 was detected by RIP assay. The results revealed that the specific adsorption level of MAFG-AS1 on Ago2 increased obviously compared to the IgG group of Huh7/R and HepG2/R cell lines (Figure 3E). We also performed RT-PCR analysis for the expression of miR-3196 in Huh7/R and HepG2/R cell lines

${ }^{1}$ http://starbase.sysu.edu.cn/mirLncRNA 
A

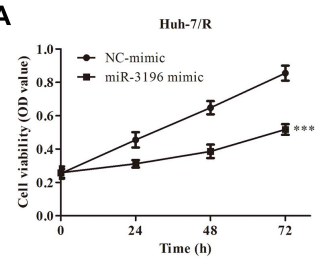

C

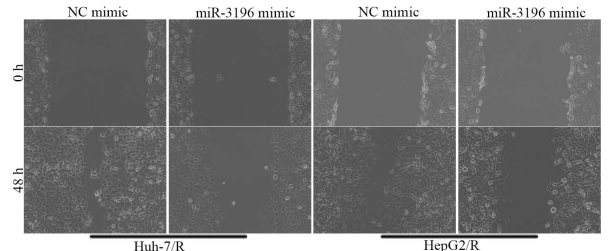

D
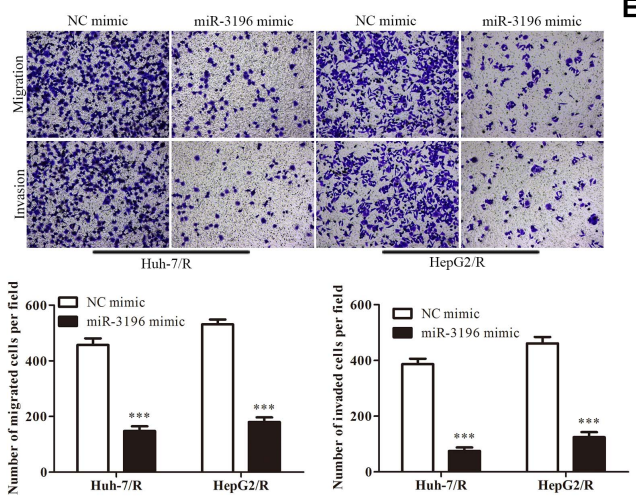

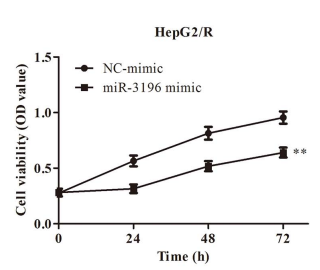

B

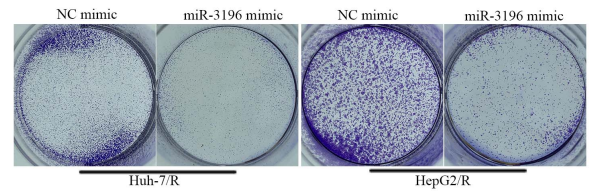

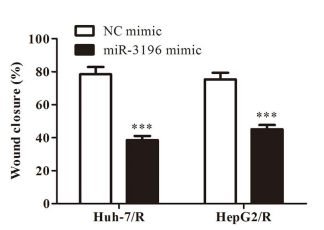

E
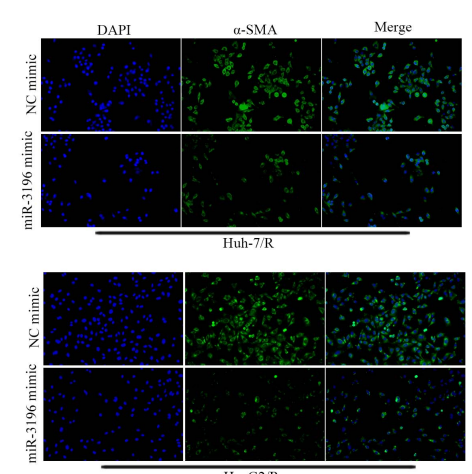

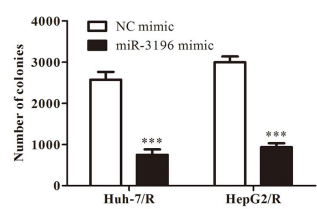

$\mathbf{F}$

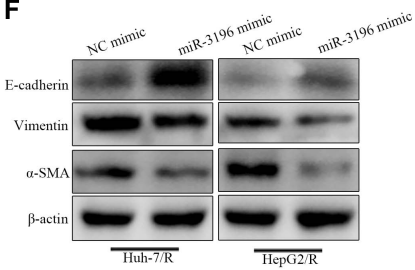

Huh- $7 / \mathbf{R}$

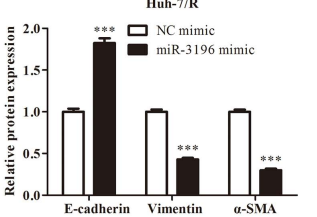

HepG $2 / \mathbf{R}$

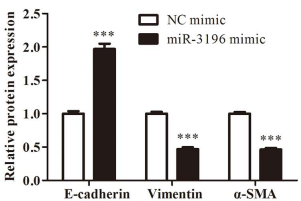

FIGURE 4 | miR-3196 overexpression inhibits proliferation, invasion, migration, and EMT of HCC cells. (A) CCK-8 assay indicated the cell viability in Huh7/R and HepG2/R cells transfected with miR-3196 mimic compared to a negative control. (B) The colony formation abilities of the Huh7/R and HepG2/R cells in miR-3196 mimic compared to the negative control. (C,D) Wound healing assay (C) and transwell analysis (D) to test the effect on the invasion and migration of HCC cells. (E,F) Immunostaining (E) and western blotting (F) detected the EMT of Huh7/R and HepG2/R cells transfected with miR-3196 mimic compared to a negative control. Scale bars: $50 \mu \mathrm{m} .{ }^{* \star} P<0.01,{ }^{* \star *} P<0.001$ vs NC-mimic group.

and parent lines, which indicated that a lower expression was detected in drug-resistant cell lines (Figure 3F). Additionally, the expression of miR-3196 was increased with MAFG-AS1 knockdown (Figure 3G). Afterward, using correlation analysis, we found a negative association between the expressions of miR3196 and MAFG-AS1 in liver cancer cells (Figure 3H). The above results demonstrate that MAFG-AS1 targets and negatively regulates $\mathrm{miR}-3196$.

\section{miR-3196 Overexpression Inhibits Proliferation, Invasion, Migration, and EMT of HCC Cells}

We further explore the effect of miR-3196 on drug-resistant liver cancer cell lines. CCK- 8 assay was used to detect the viability of cells in each group. The results showed that in contrast to the NC mimic group, the viability of Huh7/R cells in the miR-3196 mimic group was dramatically decreased. Besides, the cell viability was greatly decreased in the miR3196 mimic group of HepG2/R cell lines (Figure 4A). To further confirm the inhibition of cell proliferation by miR3196 in liver cancer cells, the colony formation assay and soft agar colony formation assay were conducted on Huh7/R and $\mathrm{HeG} 2 / \mathrm{R}$ cells. As shown in Figure $\mathbf{4 B}$, the colony formation abilities of the cells were clearly suppressed by incubation of miR-3196 mimic. Next, we performed wound healing analysis to test the effects of miR-3196 on the invasion and migration of HCC cells. The results showed that miR-3196 mimic significantly reversed liver cancer cell migration (Figure 4C). Transwell assay showed that in Huh7/R and HepG2/R cells, miR-3196 overexpression decreased the invasive cell number compared to empty vector-transfected cells (Figure 4D). Next, we investigated the induction capacity of miR-3196 to EMT in HCC cells. The results showed that overexpression of miR-3196 could 
A

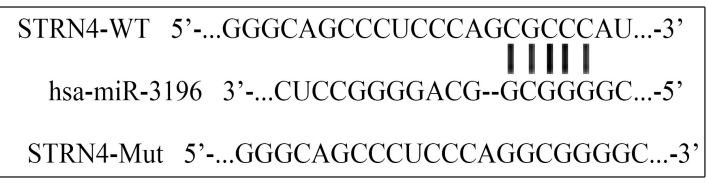

C

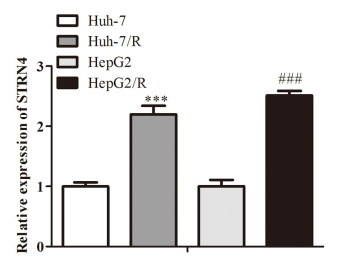

$\mathbf{F}$

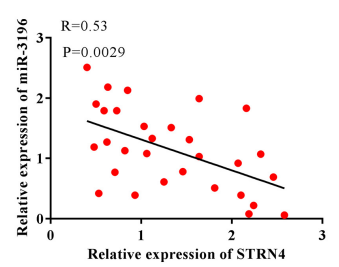

B

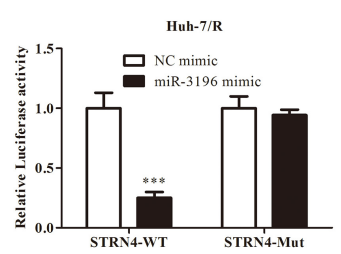

E

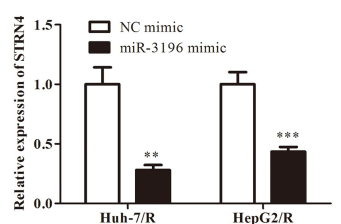

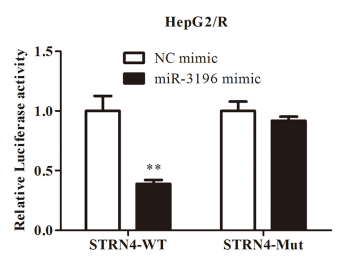

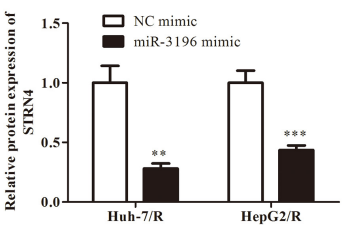

FIGURE 5 | MAFG-AS1 modulates STRN4 protein expression through targeting miR-3196 in liver cancer cells. (A) Schematic diagram shows the binding sites within miR-3196 and STRN4 mRNA 3'-UTR. (B) Luciferase reporter assay shows the molecular bond within miR-3196 and STRN4 mRNA 3'-UTR. (C,D) RT-PCR shows the STRN4 mRNA expression levels in Huh7/R and HepG2/R cells (C) or transfected with miR-3196 mimic (D). (E) Western blot analysis quantified STRN4 protein expression in Huh7/R and HepG2/R cells transfected with miR-3196 mimic and negative control mimic. (F) Correlation analysis found a negative association between the expressions of miR-3196 and STRN4 in liver cancer cells. ${ }^{\star \star} P<0.01,{ }^{\star \star \star} P<0.001,{ }^{\# \# \#} P<0.001$ vs Corresponding control group.

significantly increase the expression of E-cadherin but decrease the expression of vimentin and $\alpha$-SMA in Huh7/R and HepG2/R cells (Figures 4E,F). Overall, results concluded that miR-3196 overexpression inhibited the migration, invasion, and tumor growth of drug-resistant HCC cells, suggesting the potential tumor-promoting role of miR-3196 in drug resistance of HCC.

\section{MAFG-AS1 Modulates STRN4 Protein Expression Through Targeting miR-3196 in Liver Cancer Cells}

Next, bioinformatics results showed that miR-3196 shared complementary binding sites with STRN4 mRNA 3'-UTR (Figure 5A). Luciferase reporter assay showed that the luciferase activity was decreased when co-transfected with miR-3196 mimics and the STRN4 mild type, suggesting the molecular bond within miR-3196 and STRN4 mRNA 3'-UTR (Figure 5B). We also performed RT-PCR analysis for the expression of STRN4 in Huh7/R and HepG2/R cell lines and parent lines, which indicated a lower expression in drug-resistant cell lines (Figure 5C). In Huh7/R and HepG2/R cells, the STRN4 mRNA expression level decreased when transfected with miR3196 mimic. Additionally, Western blot revealed that miR-3196 mimic transfection decreased the STRN4 protein expression (Figures 5D,E). Additionally, correlation analysis found a negative association between the expressions of miR-3196 and STRN4 in liver cancer cells (Figure 5F). Overall, results indicated that SRTN4 acted as the target protein of miR-3196, suggesting the regulation of MAFG-AS1 on SRTN4 through targeting miR-3196.

\section{The Mechanism of MAFG-AS1 Modulating STRN4 Protein Expression Through Targeting miR-3196 in Liver Cancer Cells}

In order to investigate whether MAFG-AS1 affects the drugresistant liver cancer cell lines by regulating the miR-3196/STRN4 pathway, we manipulated the expression of miR-3196 and STRN4 with miR-3196 inhibitor and sh-STRN4, respectively. The knockdown efficiency of miR-3196 inhibitor and STRN4 shRNA-transfected HCC cells was detected by RT-qPCR. Next, CCK-8 analysis showed that sh-STRN4 decreased the activation of Huh7/R cell proliferation induced by a miR-3196 inhibitor (Figure 6A). Meanwhile, colony formation assay analysis showed that the miR-3196 inhibitor reversed Huh7/R cell proliferation by knockdown of MAFG-AS1 (Figure 6B). In addition, wound healing analysis was conducted to test the effect on the invasion and migration of HCC cells. The results showed that the miR-3196 inhibitor significantly reversed liver cancer cell migration, while STRN4 knockdown enhanced this effect (Figure 6C). Next, transwell analysis showed that the migration and invasion ability of Huh7/R and HepG2/R cells increased when these cells were co-transfected with the miR-3196 inhibitor compared with the sh-MAFG-AS1 group. Sh-STRN4 enhanced the reduction in migration and invasion induced by miR-3196 
A

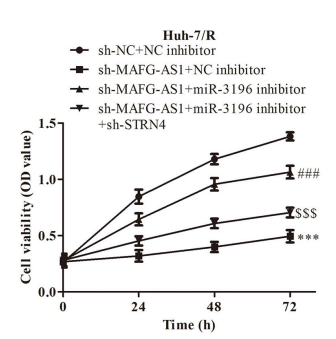

C

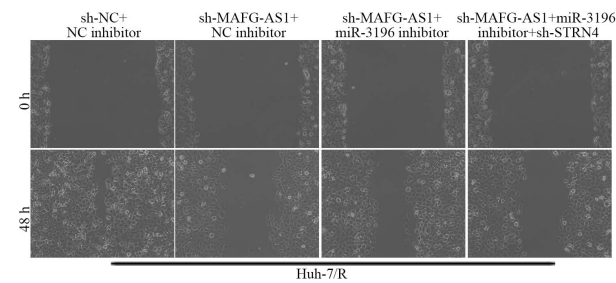

D
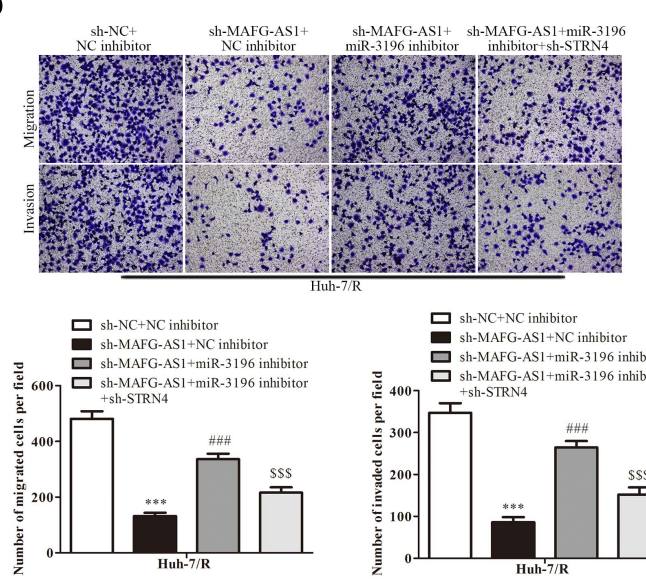

B

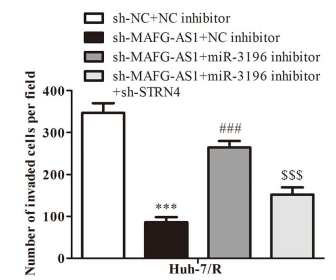

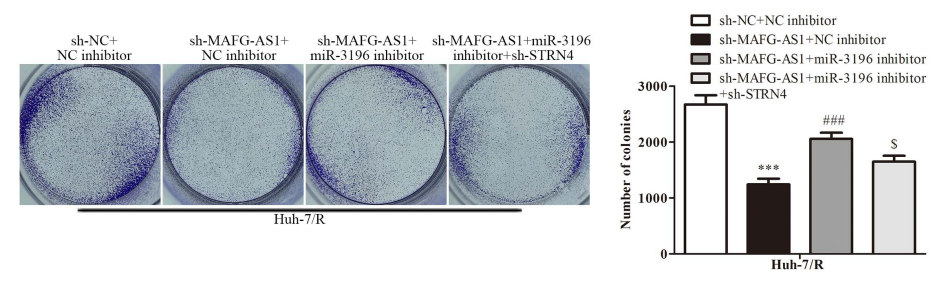

E $\quad$ sh-NC+
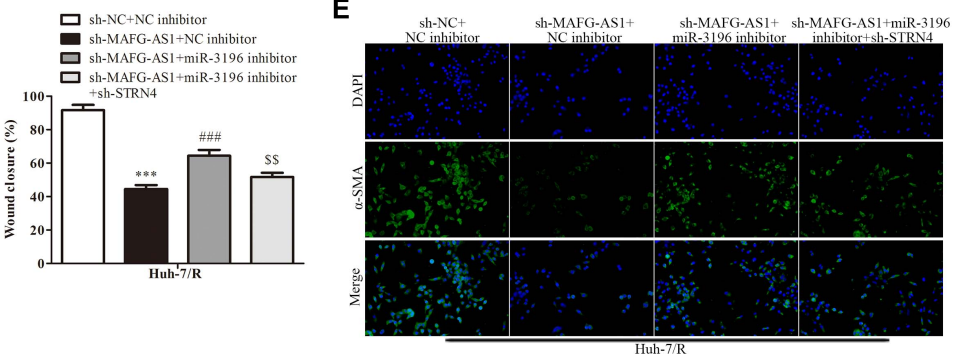

$\mathbf{F}$

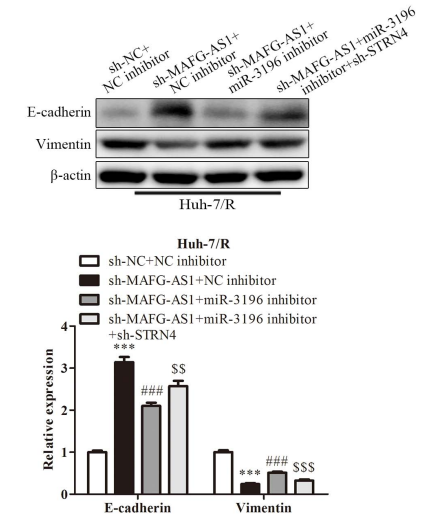

FIGURE 6 | The mechanism for MAFG-AS1 modulating STRN4 protein expression through targeting miR-3196 in liver cancer cells. (A) CCK-8 assay indicated the cell viability in Huh7/R and HepG2/R cells transfected with sh-MAFG-AS1, miR-3196 inhibitor, or sh-STRN4 compared to their negative controls. (B) The clone formation abilities of the Huh7/R and HepG2/R cells in different groups. (C) Wound healing assay (D) and transwell analysis to test the effect on the invasion and migration of HCC cells. (E) Immunostaining and (F) western blotting detected the EMT of Huh7/R and HepG2/R cells transfected with sh-MAFG-AS1, miR-3196 inhibitor, or sh-STRN4 compared to their negative controls. Scale bars: $50 \mu \mathrm{m} .{ }^{* \star *} P<0.001$ vs sh-NC + NC inhibitor group, ${ }^{\# \# \# ~} P<0.001$ vs sh-MAFG-AS1 + NC inhibitor group, $\$ P<0.05, \$ P<0.01, \$ \$ P<0.001$ vs sh-MAFG-AS1 + miR-3196 inhibitor group.

inhibitors (Figure 6D). In addition, our results showed that decreased $\alpha$-SMA proteins were induced by sh-MAFG-AS1 in Huh7/R cells, while the miR-3196 inhibitor reversed this effect (Figure 6E). The results showed that knockdown of MAFGAS1 could significantly increase the expression of E-cadherin but decrease the expression of vimentin and $\alpha$-SMA in Huh7/R and HepG2/R cells as demonstrated by IF or western blotting (Figure 6F). Based on the results, we concluded that MAFGAS1 regulates the progression of liver cancer cells through the miR-3196/STRN4 axis.

\section{DISCUSSION}

Numerous lncRNAs are aberrantly expressed in HCC, regulating various miRNAs and genes and modulating a variety of biological processes (Chen et al., 2016; She et al., 2016;
Han et al., 2017). Therefore, one or more of these lncRNAs may serve as a potential therapeutic target for treating patients with HCC. In the present study, the functional effects and potential underlying mechanism of lncRNA MAFG-AS1 in HCC were examined. Based on the RT-qPCR results, lncRNA MAFG-AS1 was highly expressed in HCC cell lines. After knockdown of lncRNA MAFG-AS1, the proliferation, migration, and invasion of HCC cell lines were significantly decreased. Interestingly, lncRNA MAFG-AS1 and miR-3196 were demonstrated to exhibit a reciprocally negative regulatory association with each other. By inhibiting the expression of miR3196, MAFG-AS1 promoted the proliferation, migration, and invasion of HCC cells.

Zhang et al. (2018) processed a regulatory network analysis of lncRNAs in colorectal cancer and showed that lncRNA MAFGAS1 was upregulated in this disease. In addition, high-throughput data analysis and in vitro experiments confirmed that lncRNA 
MAFG-AS1 was highly expressed and affects the proliferation of osteosarcoma cells. Similarly, lncRNA MAFG-AS1 expression was also upregulated in HCC cell lines and primarily distributed in the cytoplasm of HCC cells in the present study. After knockdown of lncRNA MAFG-AS1, the proliferation, migration, and invasion of HCC cell lines were significantly inhibited. Therefore, lncRNA MAFG-AS1 may serve a critical role in the pathogenesis of HCC. This study showed similar results.

Previous studies confirmed the influence of MiRNA in tumor development. Wang et al. suggested that miR-183 promoted MDR in HCC cells by regulating the miR-183IDH2/SOCS6-HIF-1 $\alpha$ feedback loop. Both miR-183 knockdown and SOCS6 overexpression sensitized BEL-7402/5-FU cells to 5-FU (Wang et al., 2016). In this study, we found that miR-3196 could bind with MAFG-AS1. MiR-3196 was lowly expressed in HCC cell lines, and the upregulation of miR-3196 repressed HCC cell proliferative and migratory capacities. Moreover, many studies proved that miRNAs can bind to the $3^{\prime}$ UTR of mRNAs to silence these mRNAs (Tomimaru et al., 2010; Wang et al., 2016). In this research, we confirmed that STRN4 was the downstream target of miR-3196 and demonstrated that STRN4 expression was positively regulated by MAFG-AS1 and negatively regulated by miR-3196.

\section{REFERENCES}

Altekruse, S. F., Henley, S. J., Cucinelli, J. E., and McGlynn, K. A. (2014). Changing hepatocellular carcinoma incidence and liver cancer mortality rates in the United States. Am. J. Gastroenterol. 109, 542-553. doi: 10.1038/ajg.20 14.11

Bao, H., Guo, C. G., Qiu, P. C., Zhang, X. L., Dong, Q., and Wang, Y. K. (2017). Long non-coding RNA Igf2as controls hepatocellular carcinoma progression through the ERK/MAPK signaling pathway. Oncol. Lett. 14, 2831-2837. doi: 10.3892/ol.2017.6492

Chen, Z., Yu, C., Zhan, L., Pan, Y., Chen, L., Sun, C., et al. (2016). LncRNA CRNDE promotes hepatic carcinoma cell proliferation, migration and invasion by suppressing miR-384. Am. J. Cancer Res. 6, 2299-2309.

Cui, S., Yang, X., Zhang, L., Zhao, Y., and Yan, W. (2018). LncRNA MAFGAS1 promotes the progression of colorectal cancer by sponging miR-147b and activation of NDUFA4. Biochem. Biophys. Res. Commun. 506, 251-258. doi: 10.1016/j.bbrc.2018.10.112

Dika, I. E., and Abou-Alfa, G. K. (2017). Treatment options after sorafenib failure in patients with hepatocellular carcinoma. Clin. Mol. Hepatol. 23, 273-279. doi: 10.3350/cmh.2017.0108

Duan, X. Y., Zhang, L., Fan, J. G., and Qiao, L. (2014). NAFLD leads to liver cancer: do we have sufficient evidence? Cancer Lett. 345, 230-234. doi: 10.1016/j.canlet. 2013.07.033

Feng, M., and Ho, M. (2014). Glypican-3 antibodies: a new therapeutic target for liver cancer. FEBS Lett. 588, 377-382. doi: 10.1016/j.febslet.2013.10.002

Han, F., Wang, C., Wang, Y., and Zhang, L. (2017). Long noncoding RNA ATB promotes osteosarcoma cell proliferation, migration and invasion by suppressing miR-200s. Am. J. Cancer Res. 7, 770-783.

Innes, H., Barclay, S. T., Hayes, P. C., Fraser, A., Dillon, J. F., Stanley, A., et al. (2018). The risk of hepatocellular carcinoma in cirrhotic patients with hepatitis $\mathrm{C}$ and sustained viral response: role of the treatment regimen. J. Hepatol. 68, 646-654. doi: 10.1016/j.jhep.2017.10.033

Ke, A. W., Shi, G. M., Zhou, J., Huang, X. Y., Shi, Y. H., Ding, Z. B., et al. (2011). CD151 amplifies signaling by integrin alpha6betal to PI3K and induces the epithelial-mesenchymal transition in HCC cells. Gastroenterology 140, 16291641.e15.

\section{CONCLUSION}

In conclusion, MAFG-AS1 was upregulated in HCC cell lines and could bind with miR-3196. Our data hinted that MAFGAS1 regulated HCC cell growth and migration through the miR-3196/STRN4 axis, exposing a potential neo-biomarker for diagnosis or treatment for liver cancer patients. These results provide important evidence that MAFG-AS1 may be a novel therapeutic target and a biomarker for predicting response to sorafenib treatment of HCC.

\section{DATA AVAILABILITY STATEMENT}

The raw data supporting the conclusions of this article will be made available by the authors, without undue reservation, to any qualified researcher.

\section{AUTHOR CONTRIBUTIONS}

TC conceived and designed the study and data extraction. $\mathrm{BH}$ performed the literature search. YP drafted the manuscript. All authors read and approved the final manuscript.

Lei, K., Liang, X., Gao, Y., Xu, B., Xu, Y., Li, Y., et al. (2017). Lnc-ATB contributes to gastric cancer growth through a MiR-141-3p/TGFbeta2 feedback loop. Biochem. Biophys. Res. Commun. 484, 514-521. doi: 10.1016/j.bbrc.2017. 01.094

Li, C., Wu, R., and Xing, Y. (2020). MAFG-AS1 is a novel clinical biomarker for clinical progression and unfavorable prognosis in gastric cancer[J]. Cell Cycle 19, 601-609. doi: 10.1080/15384101.2020.1728017

Li, T., Xie, J., Shen, C., Cheng, D., Shi, Y., Wu, Z., et al. (2016). Upregulation of long noncoding RNA ZEB1-AS1 promotes tumor metastasis and predicts poor prognosis in hepatocellular carcinoma. Oncogene 35, 1575-1584. doi: 10.1038/onc.2015.223

Liu, K., Wu, X., Zang, X., Huang, Z., Lin, Z., Tan, W., et al. (2017). TRAF4 regulates migration, invasion, and epithelial-mesenchymal transition via PI3K/AKT signaling in hepatocellular carcinoma. Oncol. Res. 25, 1329-1340. doi: 10.3727/ $096504017 \times 14876227286564$

Lu, G., Li, Y., Ma, Y., Lu, J., Chen, Y., and Jiang, Q. (2018). Long noncoding RNA LINC00511 contributes to breast cancer tumourigenesis and stemness by inducing the miR-185-3p/E2F1/Nanog axis. J. Exp. Clin. Cancer Res. 37:289.

Ma, J., Zeng, S., Zhang, Y., Deng, G., Qu, Y., Guo, C., et al. (2017). BMP4 promotes oxaliplatin resistance by an induction of epithelial-mesenchymal transition via MEK1/ERK/ELK1 signaling in hepatocellular carcinoma. Cancer Lett. 411, 117-129. doi: 10.1016/j.canlet.2017.09.041

Ruan, Z., Deng, H., Liang, M., Xu, Z., Lai, M., Ren, H., et al. (2020). Downregulation of long non-coding RNA MAFG-AS1 represses tumorigenesis of colorectal cancer cells through the microRNA-149-3p-dependent inhibition of HOXB8[J]. Cancer Cell Int. 20:511.

She, K., Huang, J., Zhou, H., Huang, T., Chen, G., and He, J. (2016). IncRNASNHG7 promotes the proliferation, migration and invasion and inhibits apoptosis of lung cancer cells by enhancing the FAIM2 expression. Oncol. Rep. 36, 2673-2680. doi: 10.3892/or.2016.5105

Tan, D. S. W., Chong, F. T., Leong, H. S., Toh, S. Y., Lau, D. P., Kwang, X. L., et al. (2017). Long noncoding RNA EGFR-AS1 mediates epidermal growth factor receptor addiction and modulates treatment response in squamous cell carcinoma. Nat. Med. 23, 1167-1175. doi: 10.1038/nm.4401

Tomimaru, Y., Eguchi, H., Nagano, H., Wada, H., Tomokuni, A., Kobayashi, S., et al. (2010). MicroRNA-21 induces resistance to the anti-tumour effect of 
interferon-alpha/5-fluorouracil in hepatocellular carcinoma cells. Br. J. Cancer 103, 1617-1626. doi: 10.1038/sj.bjc.6605958

Wang, X. J., Zhang, D.-L., Fu, C., Wei, B.-Z., and Li, G.-J. (2016). MiR-183 modulates multi-drug resistance in hepatocellular cancer (HCC) cells via miR183-IDH2/SOCS6-HIF-1alpha feedback loop. Eur. Rev. Med. Pharmacol. Sci. 20, 2020-2027.

Wang, Y., Zhang, Y., Yang, T., Zhao, W., Wang, N., Li, P., et al. (2017). Long noncoding RNA MALAT1 for promoting metastasis and proliferation by acting as a ceRNA of miR-144-3p in osteosarcoma cells. Oncotarget 8, 59417-59434. doi: 10.18632/oncotarget.19727

Xu, Y., Ge, Z., Zhang, E., Zuo, Q., Huang, S., Yang, N., et al. (2017). The IncRNA TUG1 modulates proliferation in trophoblast cells via epigenetic suppression of RND3. Cell Death Dis. 8:e3104. doi: 10.1038/cddis.2017.503

Yuan, J. H., Yang, F., Wang, F., Ma, J., Guo, Y., Tao, Q., et al. (2014). A long noncoding RNA activated by TGF-beta promotes the invasion-metastasis cascade in hepatocellular carcinoma. Cancer Cell 25, 666-681. doi: 10.1016/j. ccr.2014.03.010

Zhang, Y., Tao, Y., Li, Y., Zhao, J., Zhang, L., and Zhang, X. (2018). The regulatory network analysis of long noncoding RNAs in human colorectal cancer. Funct. Integr. Genomics 18, 261-275. doi: 10.1007/s10142-017-0588-2
Zhang, Z. F., Zhang, Y., Hu, D., Shi, J., Liu, J., Zhao, Z., et al. (2011). Smad interacting protein 1 as a regulator of skin fibrosis in pathological scars. Burns 37, 665-672. doi: 10.1016/j.burns.2010.12.001

Conflict of Interest: The authors declare that the research was conducted in the absence of any commercial or financial relationships that could be construed as a potential conflict of interest.

Publisher's Note: All claims expressed in this article are solely those of the authors and do not necessarily represent those of their affiliated organizations, or those of the publisher, the editors and the reviewers. Any product that may be evaluated in this article, or claim that may be made by its manufacturer, is not guaranteed or endorsed by the publisher.

Copyright $\odot 2021$ Chen, Huang and Pan. This is an open-access article distributed under the terms of the Creative Commons Attribution License (CC BY). The use, distribution or reproduction in other forums is permitted, provided the original author(s) and the copyright owner(s) are credited and that the original publication in this journal is cited, in accordance with accepted academic practice. No use, distribution or reproduction is permitted which does not comply with these terms. 